\title{
Review of Oral and Maxillofacial Surgeons' Imaging Use for Facial Trauma
}

\author{
Dayea Oh, Alyssa Zhang, Zeb McNamara, Paul Monsour
}

\author{
Department of Dento-maxillofacial Radiology, School of Dentistry, The University of Queensland, Queensland, Australia
}

\begin{abstract}
Introduction: Diagnostic imaging plays a crucial role in assessing maxillofacial bone fractures, both preoperatively and postoperatively. Multi-slice computed tomography (MSCT) is the current gold standard imaging modality in maxillofacial trauma; some of its benefits include fast scanning time, large field of view, and superior hard and soft-tissue details. There are limited data in current Australian literature regarding standardized imaging protocols for maxillofacial injuries. This paper aims to evaluate the current Australian oral and maxillofacial (OMF) surgeons' preferred facial trauma imaging modalities, both pre and postoperatively and to review the current literature on diagnostic imaging use in maxillofacial trauma. Methods: A survey was created for 200 members of The Australian and New Zealand Association of OMF Surgeons. The questionnaire was primarily focused on the surgeon's preferred pre-operative and post-operative imaging modalities in four clinical scenarios: "Mandibular fractures not involving condyles," "mandibular fractures involving condyles," "maxilla only fractures," and "other midfacial bone fractures." Results: MSCT was the surgeons' most preferred pre-operative and post-operative imaging modality in all scenarios except in the "mandibular fractures not involving condyles" scenario, where two-dimensional imaging was the most preferred. Cone-beam computed tomography (CBCT) was the least preferred imaging modality overall. Conclusion: MSCT should remain as the gold standard imaging modality in pre-operative maxillofacial trauma imaging, particularly in mid-face trauma.
\end{abstract}

Key words: Cone-beam computed tomography, fracture, imaging, radiology, trauma

\section{INTRODUCTION}

Maxillofacial injuries are common sequelae of motor vehicle accidents, interpersonal violence, sporting injuries, and falls. According to Gassner et al., who evaluated 9543 facial trauma cases, the midface was the most common fracture site, accounting for $70 \%$ of all facial fractures, followed by the mandible (25\%) and the upper face (5\%). ${ }^{[1]}$ Fractures in the maxillofacial region harbor many complications such as: Facial disfigurement, loss of vision, change of occlusal relationship, and development of psychological problems. ${ }^{[2]}$

\begin{tabular}{|l|l|}
\hline \multicolumn{2}{|c|}{ Access this article online } \\
\hline \multicolumn{1}{|c|}{ Publisher } & $\begin{array}{l}\text { Website: } \\
\text { www.ijdms.in }\end{array}$ \\
\hline & DOI: 10.30954/IJDMS.1.2020.3 \\
\hline
\end{tabular}

In Australia, maxillofacial fractures are the third most common trauma in elderly people after femoral neck and upper limb fractures. ${ }^{[3]}$ Maxillofacial injuries are routinely presented in most Australian hospitals and often require prompt assessment and intervention to minimize posttrauma complications. ${ }^{[4]}$ Diagnostic imaging plays a crucial role in identifying the location and extent of fractures, as well as in reviewing fractures postoperatively. The selection of appropriate imaging aids in accurate diagnosis, while maintaining consideration for the patient's safety, following the "As Low As Reasonably Achievable" and the "As Low As Reasonably Practicable" principles. ${ }^{[5]}$

Imaging modalities routinely used to assess bony fractures of the maxillofacial region include: Various views of two-dimensional (2D) imaging, multi-slice computed tomography (MSCT), and cone-beam computed tomography (CBCT). Utilization of the latter two digital three-dimensional (3D) imaging modalities, in particular, allows surgeons to accurately locate fractures and assess

\footnotetext{
Address for Correspondence:

Dr. Dayea Oh, Level 7, School of Dentistry, The University of Queensland, 288 Herston Road, Herston 4006, Queensland, Australia. Phone: +61-7-3365-8066. E-mail: d.oh@uq.edu.au
}

Submission: 12 June 2020; Revision: 11 July 2020; Acceptance: 18 July 2020 
their relationship with adjacent vital structures with the absence of superimpositions of anatomic structures. ${ }^{[6]}$ There are limited data in current Australian literature regarding standardized imaging protocols for maxillofacial injuries, especially after CBCT was added to the Australian Medicare Benefits Schedule in 2011. This paper aims to evaluate the current Australian OMF surgeons' preferred post-trauma imaging modalities.

The objectives are to analyze the surgeons' preferred imaging modalities in various maxillofacial trauma scenarios and to evaluate the prevalence of CBCT use compared to MSCT, due to its comparable diagnostic ability at a lower radiation dosage.

\section{METHODS}

\section{Study Design}

The survey questionnaire consisted of 20 questions that were tailored to OMF surgeons practicing in various States and Territories of Australia. The questionnaire was primarily focused on the participant's preferred imaging modality, or multiple modalities, between 2D imaging (including panoramic radiographs), MSCT and CBCT, for maxillofacial trauma. There was one open-ended question to elicit the reason for choosing the specific imaging modality.

The survey was designed to be anonymous and included no sensitive questions which could potentially reveal the identity of the participant. A participant information sheet with informed consent was provided before the survey. There was no perceived physical or psychological risk involved in the study.

\section{Participant Recruitment}

The total number of registered OMF surgeons in Australia at the time of the survey was 222, according to the 2018 Australian health professional registrant data. ${ }^{[7]}$ The authors approached the Queensland branch of The Australian and New Zealand Association of OMF Surgeons (ANZOAMS) for distribution of the survey questionnaire to the 200 active Australian ANZOAMS members.

\section{Survey Distribution}

The survey questionnaire was first distributed in September 2018. An online link for the electronic survey was created using Checkbox survey software (Watertown, MA, USA) and was emailed to ANZOAMS members. The online survey was available for 6 months. In addition, 40 physical copies of the survey questionnaire were provided to the Queensland branch of ANZOAMS.

\section{Data Management}

All completed and returned hard-copy surveys were converted to digital documents and disposed of securely. The final digital data were stored on a password protected standalone computer and were accessed by the primary author.

\section{Data Analysis}

Only surveys with all questions completed by currently practicing OMF surgeons were included in data analysis $(n=48)$. Descriptive statistical analysis was performed using the JASP software program and Microsoft Excel (Microsoft, Redmond, WA, USA).

The current study conforms to the Strengthening the Reporting of Observational Studies in Epidemiology guideline for cross-sectional studies.

Ethics approval for this study was granted by The University of Queensland's Human Research Ethics Committee before commencement.

\section{RESULTS}

In total, 69 of 200 ANZOAMS members responded to the survey, and only entirely completed surveys $(n=48,24 \%)$ were included for data analysis. The surgeons' demographic details are summarized in Table 1. Most participants in this study (92\%) worked full-time (>38 h/week), and many were practicing in metropolitan areas (69\%). The average duration of practice was 15.7 (median: 13.5, with a range of $1-50$ ) years among all participating surgeons. The highest survey response rate came from surgeons with fewer than 10 years of practice (44\%), followed by surgeons with $11-20$ years of practice (27\%).

Greater than $85 \%$ of the surgeons in this study worked in more than one sector; work sectors included academia, defense force, private, and public. Most (98\%) were working in the private sector at the time of this survey, followed by the public sector $(81 \%)$.

Table 1 details OMF surgeons' imaging use for maxillofacial trauma. Of all surgical cases, $16 \%$ were trauma-related and 93\% of such cases required imaging. Greater than $50 \%$ of surgeons requested imaging to both public and private imaging clinics. As a primary trauma imaging modality, all surgeons in this study have used MSCT (100\%). 2D imaging and CBCT were used as primary trauma imaging by $85 \%$ and $31 \%$ of participating surgeons, respectively.

\section{Preferred Pre-operative and Post-operative Trauma Imaging}

The OMF surgeons in this study were provided with four different scenarios of maxillofacial trauma: "Mandibular 
fractures not involving condyles," "mandibular fractures involving condyles," "maxilla only fractures," and "other midfacial bone fractures." The surgeons' preferred preoperative and post-operative imaging modalities for each scenario are analyzed and summarized in graphs [Figures 1 and 2] and in Table 2. Near equal results were observed in both pre-operative and post-operative imaging preferences.

2D imaging, including panoramic radiographs, was the most preferred pre-operative and post-operative imaging modality (69\% and 98\%, respectively) in the "mandibular fractures not involving condyles" scenario. Multi-slice CT was the surgeons' most preferred pre-operative and postoperative imaging modality in the other three scenarios [Figures 1 and 2]. In the "other midfacial bone fractures" scenario, especially, $77 \%$ of the surgeons preferred MSCT both preoperatively and postoperatively. CBCT was the least preferred imaging modality in all scenarios, both preoperatively and postoperatively. "Better details" (44\%)

Table 1: Demographics of the survey respondents and their imaging use in maxillofacial trauma

\begin{tabular}{lc}
\hline Australian oral and maxillofacial surgeons & \\
Total number of surgeons $(n)$ & $48(100 \%)$ \\
Years of practice & \\
Mean \pm SD & $15.7( \pm 12.1)$ \\
Median & 13.5 \\
Range & Min. 1, Max. 50 \\
Hours of work & \\
Full-time (>38 h/week) & $44(92 \%)$ \\
Part-time (<38 h/week) & $4(8 \%)$ \\
Work sector & \\
Public & $39(81 \%)$ \\
Private & $47(98 \%)$ \\
Academia & $21(44 \%)$ \\
Defense force & $15(31 \%)$ \\
Work region & \\
Metropolitan & $33(69 \%)$ \\
Rural or remote & $4(8 \%)$ \\
Both & $11(23 \%)$ \\
Percentage (\%) of facial trauma in practice & \\
Mean $\pm S D$ & $16 \%( \pm 10.3)$ \\
Median & $10 \%$ \\
Primary imaging site(s) & \\
Public hospital only & \\
Private imaging clinic only & $6(13 \%)$ \\
Both public and private & $11(23 \%)$ \\
In-house, public and private & $27(56 \%)$ \\
Facial trauma requiring diagnostic imaging & $4(8 \%)$ \\
Mean $\pm S D$ & \\
Median & $93 \%( \pm 21.4)$ \\
Primary trauma imaging modality (in the past 10 years) \\
MSCT ${ }^{\ddagger}$ \\
CBCT \\
$2 D \S$ imaging including panoramic radiographs & $48(100 \%)$ \\
\hline
\end{tabular}

${ }^{\dagger}$ Multi-slice computed tomography, ${ }^{\ddagger}$ cone-beam computed tomography, stwo-dimensional imaging and "machine availability" (40\%) were popular reasons why the surgeons preferred MSCT over CBCT in this study.

Preoperatively, 2D imaging (chosen by 33 surgeons) was 17 times more preferred than CBCT (chosen by 2 surgeons) in the "mandibular fractures not involving condyles" scenario; multi-slice CT was 8 and 35 times more preferred than CBCT in "mandibular fractures involving condyles" and "maxillary fractures," respectively [Table 2]. For postoperative assessment, 2D imaging (chosen by 47 surgeons) was 47 times more preferred than CBCT (chosen by one surgeon) in "mandibular fractures not involving condyles;" MSCT was, again, 8 and 35 times more preferred than CBCT in pre-operative "mandibular fractures involving condyles" and "maxillary fractures," respectively [Table 2]. No surgeons preferred "CBCT only" imaging for pre-operative and post-operative assessment of "other midfacial bone fractures."

In this survey, the surgeons were also able to select supplemental 2D imaging to any of the selected 3D imaging modalities (MSCT and CBCT); and MSCT combined with

Table 2: Surgeons' preferred pre- and post-operative imaging modalities in different clinical scenarios

\begin{tabular}{|c|c|c|}
\hline \multicolumn{3}{|c|}{$\begin{array}{l}\text { Australian oral and maxillofacial surgeons' preferred imaging } \\
\text { modalities }(n=48)\end{array}$} \\
\hline Imaging modalities & $\begin{array}{l}\text { Pre-operative } \\
\text { imaging (\%) }\end{array}$ & $\begin{array}{l}\text { Post-operative } \\
\text { imaging (\%) }\end{array}$ \\
\hline \multicolumn{3}{|c|}{ Scenario 1. Mandibular fractures not involving the condyles } \\
\hline MSCT only & $2(4.2)$ & - \\
\hline MSCT and 2D & $6(12.5)$ & - \\
\hline MSCT and/or CBCT and 2D & $3(6.3)$ & - \\
\hline СВCT only & $2(4.2)$ & $1(2.1)$ \\
\hline CBCT and 2D & $2(4.2)$ & - \\
\hline 2D only & $33(68.8)$ & $47(97.9)$ \\
\hline \multicolumn{3}{|c|}{ Scenario 2. Mandibular fractures involving the condyles } \\
\hline MSCT only & $17(35.4)$ & $17(35.4)$ \\
\hline MSCT and 2D & $15(31.3)$ & $15(31.3)$ \\
\hline MSCT and/or CBCT & $2(4.2)$ & $2(4.2)$ \\
\hline MSCT and/or CBCT and 2D & $5(10.4)$ & $5(10.4)$ \\
\hline СВCT only & $2(4.2)$ & $2(4.2)$ \\
\hline 2D only & $7(14.6)$ & $7(14.6)$ \\
\hline \multicolumn{3}{|l|}{ Scenario 3. Maxillary fractures } \\
\hline MSCT only & $35(72.9)$ & $35(72.9)$ \\
\hline MSCT and 2D & $6(12.5)$ & $6(12.5)$ \\
\hline MSCT and/or CBCT & $3(6.3)$ & $3(6.3)$ \\
\hline MSCT and/or CBCT and 2D & $1(2.1)$ & $1(2.1)$ \\
\hline CBCT only & $1(2.1)$ & $1(2.1)$ \\
\hline 2D only & $1(2.1)$ & $1(2.1)$ \\
\hline Other (not specified) & $1(2.1)$ & $1(2.1)$ \\
\hline \multicolumn{3}{|c|}{ Scenario 4. Other midfacial bone fractures } \\
\hline MSCT only & $37(77.1)$ & $37(77.1)$ \\
\hline MSCT and 2D & $7(14.6)$ & $7(14.6)$ \\
\hline MSCT and/or CBCT & $1(2.1)$ & $1(2.1)$ \\
\hline MSCT and/or CBCT and 2D & $1(2.1)$ & $1(2.1)$ \\
\hline 2D only & $1(2.1)$ & $1(2.1)$ \\
\hline Other (MRI) & $1(2.1)$ & $1(2.1)$ \\
\hline
\end{tabular}




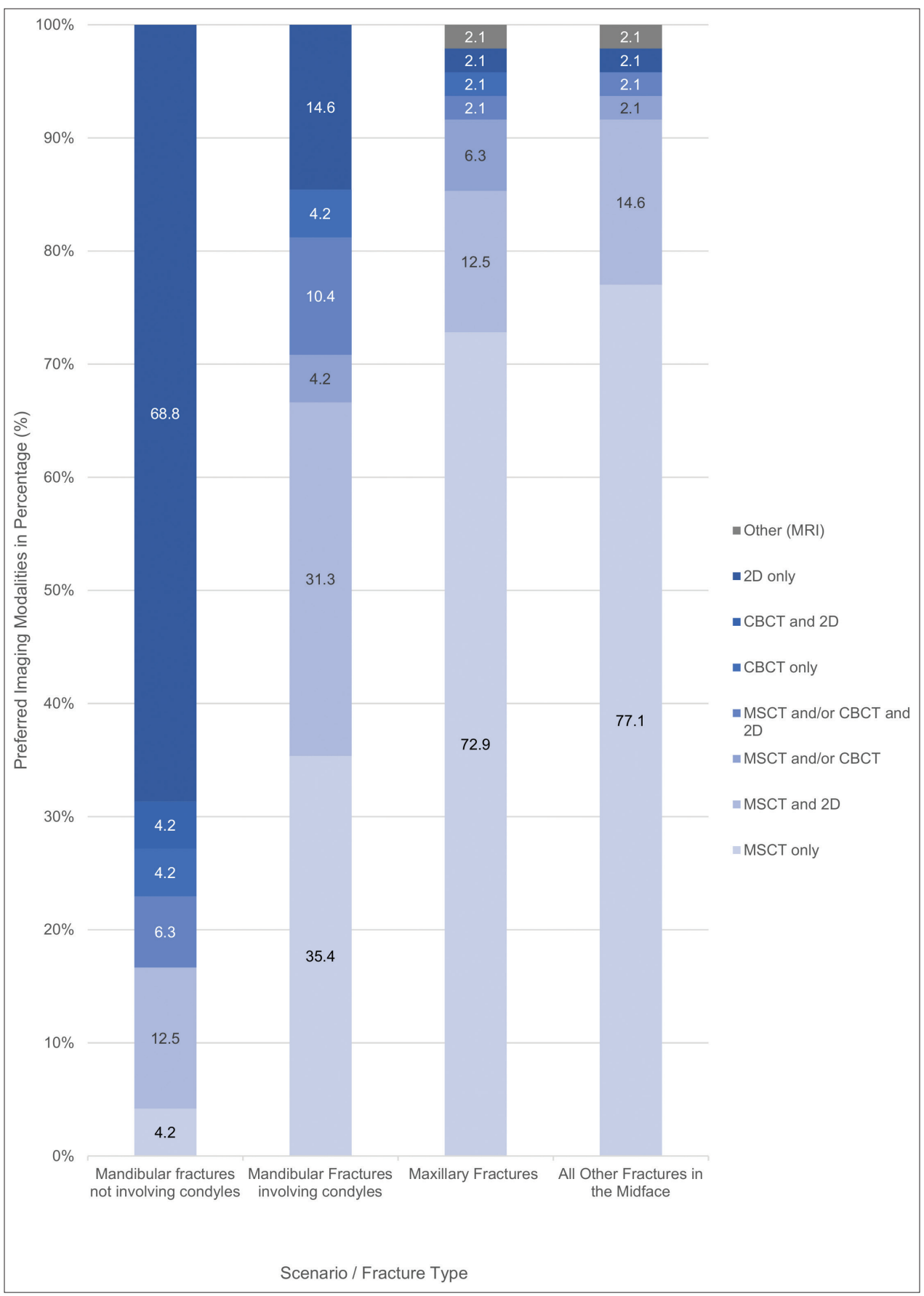

Figure 1: Stacked column graph demonstrating the surgeons' preferred pre-operative trauma imaging modalities

2D imaging, "MSCT and 2D," was the second most preferred imaging choice in all scenarios except for "mandibular fractures not involving condyles."

\section{DISCUSSION}

\section{Applications of 2D Imaging in Trauma}

Conventionally, multiple views of $2 \mathrm{D}$ conventional imaging were acquired to evaluate fractures in maxillofacial trauma. ${ }^{[8]}$
With the introduction of 3D imaging, such as CBCT and MSCT, 2D imaging is less routinely utilized in trauma. However, in zygomatic arch and mandibular fractures, it is still considered highly diagnostic and is favored by many surgeons. The current standard post-operative imaging protocol at the Royal Brisbane Women's Hospital for zygomaticomaxillary complex or zygomatic arch fracture also primarily involves 2D imaging: A combination of occipitomental (OM) 15, OM30, posteroanterior (PA) face, and submento-vertex; or, alternatively, and CT imaging. ${ }^{[9]}$ Wikner et al.'s study also state that 2D imaging is useful for diagnosing 


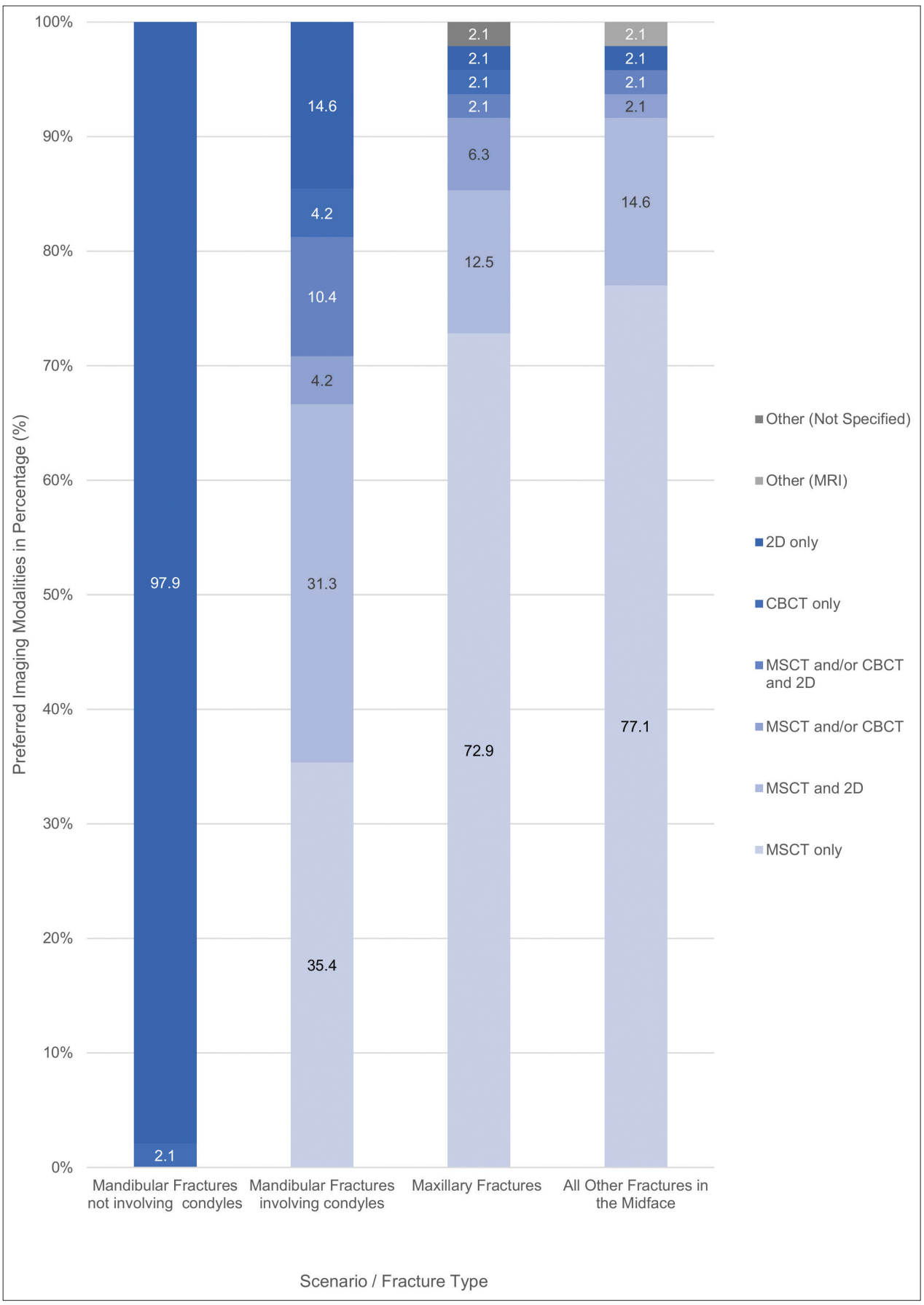

Figure 2: Stacked column graph demonstrating the surgeons' preferred post-operative trauma imaging modalities

isolated zygomatic arch fractures. ${ }^{[8]}$ For mandibular trauma without condylar fracture, the participating surgeons in this study favored 2D imaging over MSCT or CBCT, both preoperatively and postoperatively [Figures 1 and 2]. Miele and Trinci's study evaluated 2D imaging use in mandibular trauma and concluded that it was suitable for assessing fractures in the mandible, the dentoalveolar structures, but not in other facial trauma. ${ }^{[10]}$ This is supported by another study by Koshy et al. who stated that panoramic radiographs were "the best" for assessing dentition-related mandibular fractures, although CT performs the best for other fracture sites in the mandible; CT has a $100 \%$ sensitivity in detecting mandibular fractures. ${ }^{[11,12]}$

Aside from assessing the zygomatic arch and mandibular fractures, 2D imaging is not routinely used on its own. It has been reported that 2D imaging is now more often used in conjunction with 3D imaging in various maxillofacial trauma cases. ${ }^{[13,14]}$ This present survey study also supports this statement as "MSCT combined with 2D" imaging was 
the second-most preferred imaging modality after "MSCT only" imaging by surgeons for assessment of various facial bone fractures, especially in mandibular fractures involving condyles, maxilla, and other midfacial bone fractures [Figures 1 and 2]. The popularity of the "2D and 3D" imaging combination is most likely due to the surgeons' improved diagnostic capabilities with various available views by adding relatively low-dose $2 \mathrm{D}$ imaging to more comprehensive $3 \mathrm{D}$ imaging at minimal cost, time, and storage. ${ }^{[13,15]}$

\section{Gold Standard Imaging in Trauma: Multi-slice CT}

Utilization of 3D imaging modalities such as MSCT and CBCT enables detailed assessment of maxillofacial structures in various reconstructed views without superimposition of anatomical structures or image magnification. ${ }^{[16]}$ In maxillofacial trauma, MSCT is the "gold standard" imaging modality and is the most frequently used modality worldwide. ${ }^{[17]}$ The widely accepted New Orleans CT guideline in maxillofacial trauma also states that "head CT scan is the first-line examination and is recommended for any patient without loss of consciousness or posttraumatic amnesia, or if any of the following is present: Neurological deficit, vomiting, severe headache, age over 65 years, suspected skull-base fracture, Glasgow score $<15$, coagulopathy, and trauma with dangerous mechanism." ${ }^{[18]}$ In addition, MSCT is the only imaging modality which is able to scan the entire body in a supine position at an accelerated speed in polytrauma cases. ${ }^{[19]}$ The average scan time of a head CT is less than $1 \mathrm{~s}$ with multi-slice CT scanners. ${ }^{[8]}$ Its exceptional speed allows prompt assessment of fractures and swift preparation of emergency surgery. In cases of complex reconstructive surgery requiring autogenous bone grafts from other parts of the body such as calvaria, iliac crests, and tibia, MSCT is the only modality which scans the entire body without restrictions. ${ }^{[20]}$ Furthermore, MSCT imaging has superior soft-tissue contrast as windowing the greyscale image enables the viewing of both bony and soft-tissues. Soft-tissue windowing is a superb ancillary feature, especially when the facial trauma involves the naso-orbito-ethmoid fractures, facial swelling, and other associated soft-tissue injuries.

All participating OMF surgeons in this study have used MSCT as a primary imaging modality in maxillofacial trauma. It was also the surgeons' most preferred preoperative and post-operative imaging modality in most facial bone fracture types [Figures 1 and 2]. Aside from the aforementioned advantages of MSCT use for trauma imaging, the motive behind opting for MSCT is also likely associated with the longer history of MSCT use compared to CBCT and accessibility of the imaging modality. Multislice CT scanners of early generations (2-slice and 4-slice scanners) were available for general radiology since the 1990 's. ${ }^{[21]}$ In recent years, not only have MSCT scanners become more readily accessible in hospitals and radiology clinics, they have also become far more advanced (64 or more slices at isotropic voxels). ${ }^{[2]}$ According to the Organization for Economic Co-operation and Development (OECD)'s 2017 health data, the total count of CT scanners in Australia was 1583. The total number of CBCT scanners in Australia was approximately 280 (ranging from 232 to 328 ) in the same year. ${ }^{[22]}$ CBCT imaging was only added to the Australian Medicare Benefits Schedule in July 2011 as codes: 56025 and 56026. If the CT scanner count by the OECD included all MSCT scanners, there were approximately 5.7 times more MSCT scanners than CBCT scanners in Australia in 2017, somewhat justifying the disparity between MSCT and CBCT use.

The main weakness of MSCT is its highly effective radiation dose. The effective dose of the average CT head scan is $1-3 \mathrm{mSv}{ }^{[23]}$ Although there is no dose limit to medical radiation exposure in Australia, this dosage is still equal to, or greater than, the current allowable annual dose limit for the general public of $1 \mathrm{mSv}^{\left[{ }^{[2]}\right.}$ Comparatively, the average effective radiation dose of a large field of view CBCT scan $(>15 \mathrm{~cm}$ in height) ranges from $0.046 \mathrm{mSv}$ to 0.916 $\mathrm{mSv}$, depending on the type of CBCT machine. ${ }^{[25]}$ This is significantly lower than MSCT's dosage, considering CBCT is also a $3 \mathrm{D}$ imaging modality.

\section{Use of CBCT in Post-operative Imaging}

In the current study, CBCT was the least preferred preoperative and post-operative imaging modality by the participants [Figures 1 and 2]. Although CBCT may be less practical in pre-operative assessments of complex facial trauma, mainly due to its relatively lengthy scan time, nonsupine patient positioning, sensitivity to movement and poor soft-tissue image contrast; it is a suitable imaging modality for post-operative examination and for assessing mandibular trauma with or without the involvement of condyles. ${ }^{[6]}$ Sirin et al.'s study compared the accuracy of CBCT to MSCT in assessing fracture of a sheep's mandibular condyles; there was no statistically significant difference between the two imaging modalities. ${ }^{[26]}$ Kaeppler et al. analyzed the accuracy of CBCT in assessing mandibular fractures and stated, "in patients with suspected mandibular fracture, CBCT increases diagnostic certainty to $90.5 \%$." [27] Ilguy et al. invited three OMF radiologists to assess multiple dentoalveolar fractures using both MSCT and CBCT scans, and the results showed all three radiologists identifying more fractures on the CBCT imaging than on MSCT. ${ }^{[28]}$ There is evidence in the literature that CBCT sensitivity is superior in identifying small fractures in the multi-fragmented midface and mandibular fractures. ${ }^{[17]} \mathrm{CBCT}$ has a superior spatial resolution of hard tissues compared to MSCT. ${ }^{[29]}$ In a systematic review by Caruso et al., who analyzed CBCT use in temporomandibular joints, superior demonstration of hard tissue details was observed, as well as CBCT's ability to measure condylar volume and surface area. ${ }^{[30]}$ 


\section{Limitation of the Current Study}

The current study is the first to evaluate the use of diagnostic imaging modalities (between 2D, MSCT, and CBCT) in maxillofacial trauma by Australian OMF surgeons. The major limitations of the current study are: Low response rate, limited details in clinical scenarios for the survey, and reduced accessibility of CBCT compared to other imaging modalities. A follow-up, long-term study is warranted to eliminate the mitigating limitations of the current study.

\section{CONCLUSION}

Determining the most suitable imaging modality for maxillofacial trauma is significantly influenced by several factors which are mainly: The nature, severity, and extent of the trauma, and the region and types of bones involved. $2 \mathrm{D}$ imaging is useful for assessing fractures in the zygomatic arch and the mandible, but when supplemented with 3D imaging, it may be used in complicated polytrauma cases to aid surgeons in improved pre-operative diagnostic capability. Multi-slice CT is the "gold standard" trauma imaging modality, which is superior in assessing all types of maxillofacial fractures and polytrauma cases; it is able to scan large fields in short scan times, and also has superior hard and soft-tissue details. In the current study, MSCT was the most preferred imaging modality by Australian OMF surgeons in most maxillofacial trauma cases, both preoperatively and postoperatively. CBCT is a newer 3D imaging modality which was first added to the Australian Medicare Benefits Schedule in the year 2011, and it was the least preferred pre-operative and post-operative imaging modality by the surgeons. CBCT, however, demonstrates superior hard tissue details at a relatively low radiation dose, but it lacks soft-tissue details and has a longer scanning time compared to MSCT. Considering multiple factors, such as effective radiation dose and improved hard tissue resolution, CBCT may still be a viable alternative to MSCT in post-operative maxillofacial trauma assessment. A follow-up study is warranted for the assessment of future maxillofacial trauma imaging protocols.

\section{ACKNOWLEDGMENTS}

This paper was partially funded by The University of Queensland's Postgraduate Student Research Support Grant.

The authors acknowledge the assistance of the ANZOAMS with survey distribution.

\section{AUTHOR DECLARATION}

All authors: Drs Dayea Oh, Alyssa Zhang, Zeb McNamara, and Paul Monsour confirm that the manuscript has been read, gave their final approval and agree to be accountable for all aspects of the work.

The authors also declare no potential conflicts of interest with respect to the authorship and/or publication of this article.

The authors declare that all financial support that might bias the interpretation of the work described in this manuscript has been fully addressed.

Ethics approval for this study was granted by The University of Queensland's Human Research Ethics Committee before commencement (Approval Number: 2018001200).

\section{REFERENCES}

1. Gassner R, Tuli T, Hachl O, Rudisch A, Ulmer H. Craniomaxillofacial trauma: A 10 year review of 9,543 cases with 21,067 injuries. J Craniofac Surg 2003;31:51-61.

2. De Sousa A. Psychological issues in acquired facial trauma. Indian J Plast Surg 2010;43:200-5.

3. Cripps R, Carman J. Falls by the Elderly in Australia: Trends and Data for 1998. Australia: Australian Institiute of Health and Welfare; 2001.

4. Lynham A, Tuckett J, Warnke P. Maxillofacial trauma. Aust Fam Physician 2012;41:172-82.

5. Grange S. Vital guide to radiography and radiation protection. Vital 2009; 7:43-6.

6. Weiss $\mathrm{R} 2^{\text {nd }}$, Read-Fuller A. Cone beam computed tomography in oral and maxillofacial surgery: An evidence-based review. Dent J (Basel) 2019;7:52.

7. Dental Board of Australia. Dental Board of Australia. Registration Data Table. Dental Board of Australia. Available from: http:// www.dental-board.gov.au/About-the-Board/Statistics.aspx. [Last accessed on 2019 Apr 22].

8. Wikner J, Riecke B, Gröbe A, Heiland M, Hanken H. Imaging of the midfacial and orbital trauma. Facial Plast Surg 2014;30:528-36.

9. Higgins A, Hurrell M, Harris R, Findlay G, David M, Batstone M. A study protocol for a randomised controlled trial evaluating the effects of intraoperative computed tomography on the outcomes of zygomatic fractures. Trials 2019;20:514.

10. Miele V, Trinci M. Diagnostic Imaging in Polytrauma Patients. $1^{\text {st }}$ ed. Cham: Springer; 2018.

11. Koshy J, Feldman E, Chike-Obi C, Bullocks J. Pearls of mandibular trauma management. Semin Plast Surg 2010;24:357-74.

12. Wilson I, Lokeh A, Benjamin C, Hilger PA, Hamlar DD, Ondrey FG, et al. Prospective comparison of panoramic tomography (zonography) and helical computed tomography in the diagnosis and operative management of mandibular fractures. Plast Reconstr Surg 2001;107:1369-75.

13. Langner S. Optimized imaging of the midface and orbits. GMS Curr Top Otorhinolaryngol Head Neck Surg 2015;14:1-24.

14. Shintaku W, Venturin J, Azevedo B, Noujeim M. Applications of cone-beam computed tomography in fractures of the maxillofacial complex. Dent Traumatol 2009;25:358-66.

15. Mesgarani A, Haghanifar S, Ehsani M, Yaghub S, Bijani A. Accuracy of conventional and digital radiography in detecting external root resorption. Iran Endod J 2014;9:241-5.

16. Yatabe M, Prieto JC, Styner M, Zhu H, Ruellas AC, Paniagua B, et al. 3D superimposition of craniofacial imaging-the utility of multicentre collaborations. Orthod Craniofac Res 2019;22:213-20.

17. Sitzman T, Sillah N, Hanson S, Gentry L, Doyle J, Gutowski K. 
Validation of clinical criteria for obtaining maxillofacial computed tomography in patients with trauma. J Craniofac Surg 2015;26:1199202.

18. Smits MD, De Haan D, Dekker G, Vos H, Kool P, Nederkoorn D, et al. External validation of the Canadian CT head rule and the new orleans criteria for CT scanning in patients with minor head injury. JAMA 2005;294:1511-8.

19. Myga-Porosiło J, Skrzelewski S, Sraga W, Borowiak H, Jackowska Z, Kluczewska E. CT Imaging of facial trauma. Role of different types of reconstruction. Part I-bones. Pol J Radiol 2011;76:41.

20. Elsalanty M, Genecov D. Bone grafts in craniofacial surgery. Craniomaxillofac Trauma Reconstr 2009;2:125-34.

21. Kohl G. The evolution and state-of-the-art principles of multislice computed tomography. Proc Am Thorac Soc 2005;2:470-6.

22. Zhang AC, Critchley S, Monsour PA. Comparative adoption of cone beam computed tomography and panoramic radiography machines across Australia. Aust Dent J 2016;64:489-96.

23. Ludlow J, Ivanovic M. Comparative dosimetry of dental CBCT devices and 64-slice CT for oral and maxillofacial radiology. Oral Surg Oral Med Oral Pathol Oral Radiol Endod 2008;106:106-14.

24. Australian Radiation Protection and Nuclear Safety Agency. Australian Radiation Protection and Nuclear Safety Agency. Recommendations for Limiting Exposure to Ionizing Radiation and National Standard for Limiting Occupational Exposure to Ionizing Radiation. Dental Board of Australia: Australian Radiation Protection and Nuclear Safety Agency. Available from: https:// www.safeworkaustralia.gov.au/. [Last accessed on 2019 Apr 22].
25. Ludlow J, Timothy R, Walker C, Hunter R, Benavides E, Samuelson DB, et al. Effective dose of dental CBCT-a meta analysis of published data and additional data for nine CBCT units. Dento maxillo facial radiology. Dentomaxillofac Radiol 2015;44:1-25.

26. Sirin Y, Guven K, Horasan S, Sencan S. Diagnostic accuracy of cone beam computed tomography and conventional multislice spiral tomography in sheep mandibular condyle fractures. Dentomaxillofac Radiol 2010;39:336-42.

27. Kaeppler G, Cornelius CP, Ehrenfeld M, Mast G. Diagnostic efficacy of cone-beam computed tomography for mandibular fractures. Oral Surg Oral Med Oral Pathol Oral Radiol 2013;116:98-104.

28. Ilguy D, Ilguy M, Fisekcioglu E, Bayirli G. Detection of jaw and root fractures using cone beam computed tomography: A case report. Dentomaxillofac Radiol 2009;38:169-73.

29. Lechuga L, Weidlich GA. Cone beam CT vs. fan beam CT: A comparison of image quality and dose delivered between two differing CT imaging modalities. Cureus 2016;8:778.

30. Caruso S, Storti E, Nota A, Ehsani S, Gatto R. Temporomandibular joint anatomy assessed by CBCT images. Biomed Res Int 2017;2017:2916953.

How to cite this article: Oh D, Zhang A, McNamara Z, Monsour P. Review of Oral and Maxillofacial Surgeons' Imaging Used for Facial Trauma. Int J Dent Med Spec 2020;7(1):7-14.

Source of Support: None; Conflicts of Interest: None 